TITLE:

\title{
Measurement of internal magnetic flux density distribution in air-core toroidal transformer under high- frequency excitation
}

\section{AUTHOR(S):}

Hashimoto, Kazuki; Okuda, Takafumi; Hikihara, Takashi

\section{CITATION:}

Hashimoto, Kazuki ... [et al]. Measurement of internal magnetic flux density distribution in air-core toroidal transformer under high-frequency excitation. Review of Scientific Instruments 2020, 91(4): 044703.

\section{ISSUE DATE:}

2020-04-01

URL:

http://hdl.handle.net/2433/252391

\section{RIGHT:}

This article may be downloaded for personal use only. Any other use requires prior permission of the author and AIP Publishing. This article appeared in 'Review of Scientific Instruments' 91, 044703 (2020); https://doi.org/10.1063/1.5123774 and may be found at https://aip.scitation.org/doi/10.1063/1.5123774.; The full-text file will be made open to the public on 02 April 2021 in accordance with publisher's 'Terms and Conditions for SelfArchiving' 


\title{
Measurement of internal magnetic flux density distribution in air-core toroidal transformer under high-frequency excitation
}

Cite as: Rev. Sci. Instrum. 91, 044703 (2020); doi: 10.1063/1.5123774

Submitted: 9 August 2019 - Accepted: 14 March 2020 •

Published Online: 2 April 2020

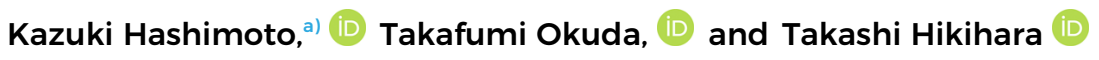

AFFILIATIONS

Department of Electrical Engineering, Kyoto University, Katsura, Nishikyo, Kyoto 615-8510, Japan

a) Author to whom correspondence should be addressed: k-hashimoto@dove.kuee.kyoto-u.ac.jp

\begin{abstract}
An investigation of air-core toroidal transformers has been undertaken to enable power converters to achieve megahertz operation. The characteristics of the air-core toroidal transformer govern the behaviors of the internal magnetic flux of the transformer. Understanding the behaviors at an above-megahertz level is a key issue in the design of the air-core toroidal transformer. This paper discusses the internal magnetic flux density distribution in the air-core toroidal transformer under high-frequency excitation. We propose a measurement method for the distribution and show the obtained results under a sinusoidal excitation with an amplitude of $1.5 \mathrm{~A}$ and a frequency of $1 \mathrm{MHz}$. The results reveal a non-uniform distribution in the tangential and normal directions. The cause of this non-uniformity was found to be the structure of the air-core toroidal transformer. In addition, the validity of the proposed measurement method was confirmed by comparing the experimentally obtained results with a numerical estimation using the Biot-Savart law. These results suggest that the proposed measurement method is capable of investigating the distributed characteristics of air-core toroidal transformers under megahertz excitation.
\end{abstract}

Published under license by AIP Publishing. https://doi.org/10.1063/1.5123774

\section{INTRODUCTION}

Megahertz operation of power converters has been investigated. ${ }^{1-3}$ As the switching frequency increases, the passive components in power converters can be miniaturized. ${ }^{4}$ Silicon carbide (SiC) metal-oxide-semiconductor field-effect transistors (MOSFETs) are suitable for megahertz operation of power converters because $\mathrm{SiC}$ MOSFETs can operate faster than silicon $(\mathrm{Si})$ power devices with the same withstand voltage. ${ }^{5-7}$ Due to highfrequency gate driver development, $\mathrm{SiC}$ MOSFETs can operate up to $10 \mathrm{MHz}$.

Still, the magnetic components such as transformers and inductors present difficult obstacles in achieving megahertz operation. One of the issues is the frequency response of magnetic materials such as $\mathrm{Mn}-\mathrm{Zn}$ and $\mathrm{Ni}-\mathrm{Zn}$ ferrite above $1 \mathrm{MHz}$. Conventional magnetic components employ magnetic materials of high permeability, in order to confine the magnetic flux. However, they cannot be utilized in the megahertz-class operation because their iron losses are not practically negligible above $1 \mathrm{MHz}{ }^{4}$ An air-core configuration is an alternative solution for megahertz-class power converters. $^{9-12}$ Air-core magnetic components are free from magnetic material iron losses. However, their magnetic flux density in the high-frequency region must be considered due to the following reasons.

The magnetic flux density is determined by the current and permeability. In the air-core configuration, the magnetic flux density depends mainly on the current because the permeability is uniform. The current flows solely along the windings of the air-core magnetic components when the excitation frequency is below the self-resonant frequency. However, when the excitation frequency is higher than the self-resonant frequency, the current flows not only along the windings but also through the stray capacitances distributed between the windings. A previous study reported that in a toroidal inductor, the current flowing through the parasitic capacitances changes the external magnetic flux density. ${ }^{13}$ Therefore, the internal magnetic flux density also changes. The internal magnetic flux density is a key governing factor for the performance of the magnetic components. Parasitic capacitances appear to have strong 
impacts during switching transients of power converters. However, it is difficult to numerically investigate the internal magnetic flux density under high-frequency switching conditions due to the computational cost. Therefore, experimental investigations are necessary in order to achieve the megahertz operation.

There have been experimental studies on the internal magnetic flux density distribution of the magnetic components used in the below-kilohertz frequency range. ${ }^{14-16}$ However, few reports are available on the frequency range over megahertz. In the investigations under the kilohertz level, the number of measurement points is limited because the magnetic components use the magnetic materials. The air-core configurations allow the magnetic sensor to be installed easily inside the air-core magnetic components. However, it is not clear whether the internal magnetic flux density of the air-core magnetic components can be measured with a high spatial resolution for frequencies over the megahertz range.

In this paper, we study an air-core transformer with a toroidal topology, which is suitable for confining the magnetic flux. We propose a measurement method for the internal magnetic flux density distribution. Then, we present the measured magnetic flux density at $1 \mathrm{MHz}$ excitation in order to demonstrate the effectiveness of the proposed method. The obtained magnetic flux densities are discussed in terms of their relationship to the structure of the air-core toroidal transformer.

\section{EXPERIMENTAL SETUP}

This section addresses the measurement method for the internal magnetic flux density distribution in an air-core toroidal transformer. Figure 1 shows the experimental setup schematic for measuring the internal magnetic flux density.
A sinusoidal current is fed to the air-core toroidal transformer as an excitation current. The excitation current is generated with a function generator (NF CORPORATION, WF1968), a bipolar power supply (NF CORPORATION, HSA4011), and an $L C$ filter. The bipolar power supply amplifies the signal from the function generator; however, the amplified signal is slightly distorted because of the characteristics of the bipolar power supply. Therefore, an $L C$ filter was inserted between the bipolar power supply and the air-core toroidal transformer to remove the distortion in the amplified signal. The generated excitation current $i_{\mathrm{p}}$ is then fed to the primary side of the air-core toroidal transformer.

The magnetic flux densities are measured with search coils and an IsoVu isolated probe (Tektronix, TIVH08). When the excitation current $i_{\mathrm{p}}$ is fed to the primary side, a magnetic flux arises in the air-core toroidal transformer. The search coil voltage is induced by the variation in the magnetic flux passing through the search coil cross section. Assuming that the search coil is sufficiently smaller in cross section than the air-core toroidal transformer, the relationship between the voltage of the search coil $v_{\text {search }}$ and the magnetic flux density $B$ can be estimated by Eq. (1),

$$
B=\frac{1}{\mathrm{AT}} \int v_{\text {search }} \mathrm{d} t .
$$

The area-turn product, denoted as AT, is determined from the number of turns and the cross sectional area of the search coil. The number of turns in the search coil is set to six turns and the cross section to $9.6 \mathrm{~mm}^{2}$. We determine the area-turn product from these values. The cross section of the search coil satisfies the previously stated assumption because it has a value less than $1 / 10$ of that of the transformer. Then, the internal magnetic flux density $B$ can be obtained from the measured voltage $v_{\text {search }}$. We fabricate two kinds of search coils for magnetic flux density measurement: tangential

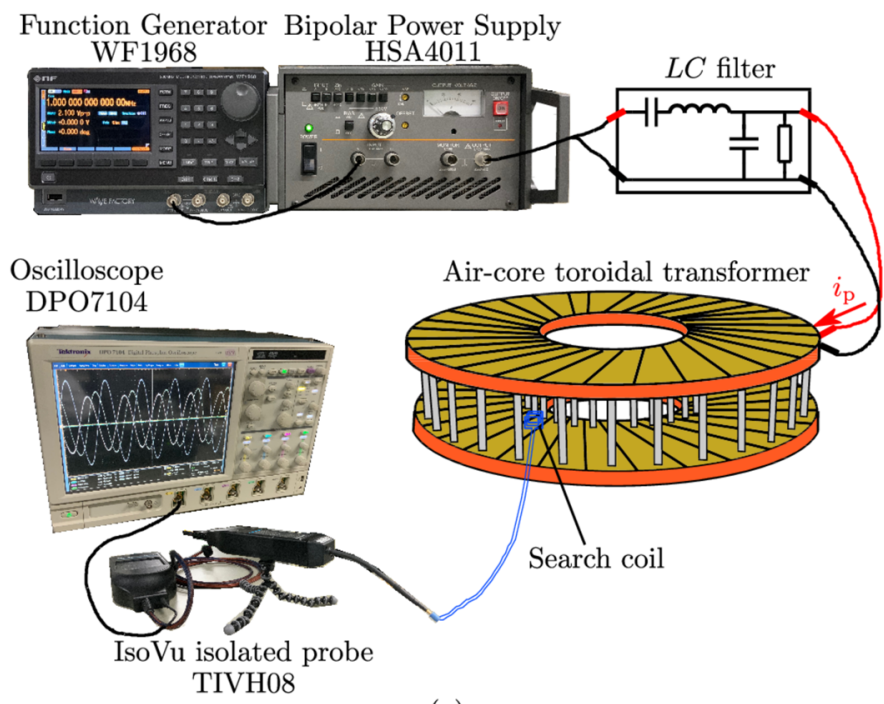

(a)

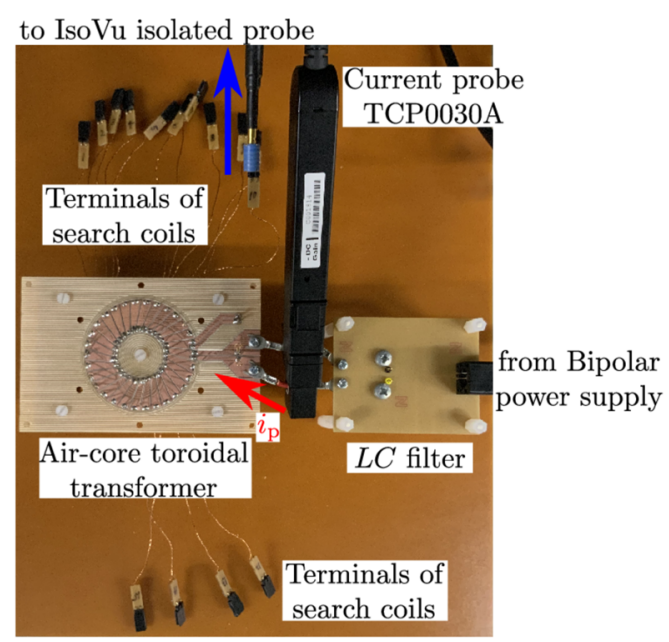

(b)

FIG. 1. Experimental setup for measuring internal magnetic flux density distribution in the air-core toroidal transformer: (a) measurement setup schematic and (b) photograph of the equipment surrounding the air-core toroidal transformer. 


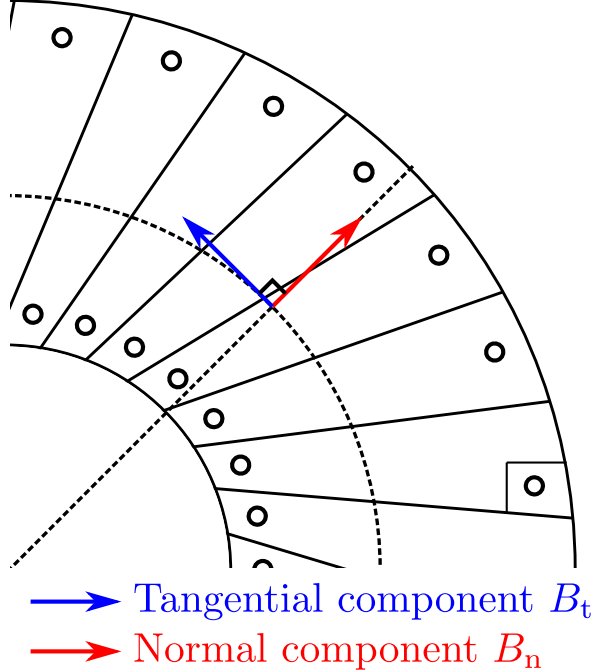

FIG. 2. Definition of tangential and normal components.

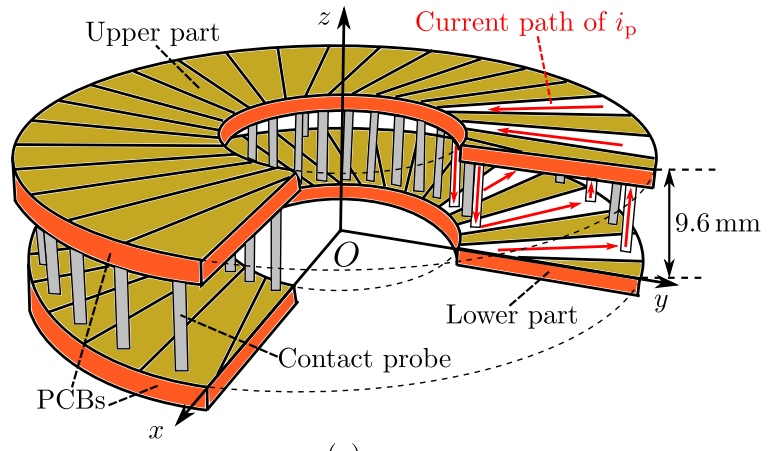

(a)

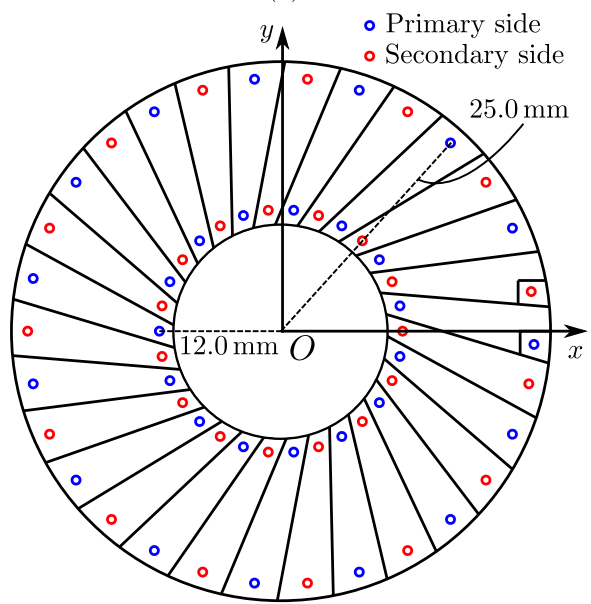

(b)

FIG. 3. Schematics of the air-core toroidal transformer: (a) overall diagram and (b) top view of the upper part. Blue circles denote primary side contact probes and red circles denote secondary side contact probes. and normal components, as defined in Fig. 2. The voltage of the search coil $v_{\text {search }}$ is measured by using an Iso $\mathrm{Vu}$ isolated probe and an oscilloscope (Tektronix, DPO7104). The magnetic flux density $B$ is obtained by integrating $v_{\text {search }}$ numerically by the trapezoidal rule.

We additionally fabricate the air-core toroidal transformer. Figures 3 and 4 show the schematics and photographs of the air-core toroidal transformer. The air-core toroidal transformer is fabricated using printed circuit boards (PCBs) and contact probes. The inner diameter is set to $12.0 \mathrm{~mm}$ and the outer diameter to $25.0 \mathrm{~mm}$. The height of the air-core toroidal transformer is $9.6 \mathrm{~mm}$. The number of turns in both the primary and secondary windings is fixed at 15 turns each.

The details of the experimental method are shown below. The purpose of this study is to demonstrate the effectiveness of the proposed method in the megahertz range. The sensitivity of the search coils is proportional to the excitation frequency. The fabricated search coils cannot measure magnetic flux densities in the kilohertz range. These coils also cannot measure the magnetic flux density in the frequency range near their self-resonant frequency. The self-resonant frequency of the fabricated search coils is higher than $100 \mathrm{MHz}$. The validity of the measurement results at $1 \mathrm{MHz}$ implies the validity of the measurement results from $1 \mathrm{MHz}$ to $100 \mathrm{MHz}$. The detailed discussion is shown in Appendix A. Therefore, an excitation current $i_{\mathrm{p}}$ with an amplitude of $1.5 \mathrm{~A}$ and a frequency of $1 \mathrm{MHz}$ is fed into the air-core toroidal transformer. We measure the magnetic flux density $B$ for four cycles. The search coils are located at the middle height of the air-core toroidal transformer $(z=4.8 \mathrm{~mm})$. Figure 5 shows the measurement points used in this study. The sampling frequency of the oscilloscope is set to $5 \mathrm{GHz}$, and the excitation current $i_{\mathrm{p}}$ is measured with a current probe (Tektronix, TCP0030A).

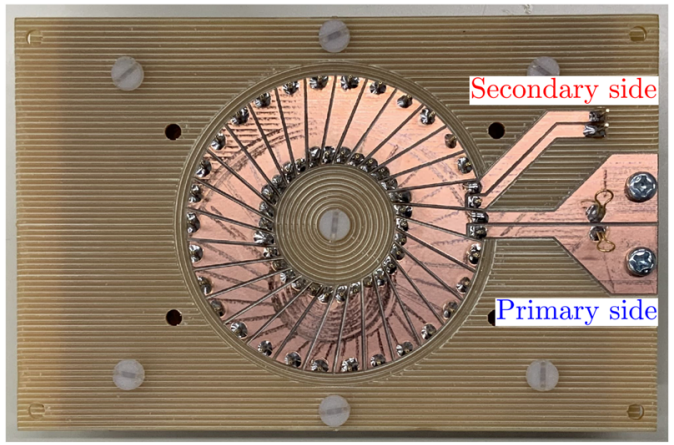

(a)

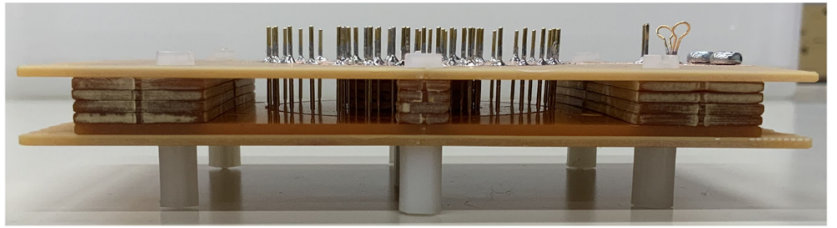

(b)

FIG. 4. Photographs of the air-core toroidal transformer: (a) top view and (b) side view. 




FIG. 5. Measurement points in the air-core toroidal transformer. Numbers around the circle denote angle. Blue circles denote primary side contact probes and red circles denote secondary side contact probes.

\section{RESULTS AND DISCUSSION}

\section{A. Internal magnetic flux density distribution in the air-core toroidal transformer}

This subsection presents the experimental results of the internal magnetic flux density distribution for the air-core toroidal transformer. First, we discuss the magnetic fluxes measured at point A in Fig. 5 .

Figure 6 shows the waveforms of (a) the excitation current, (b) the search coil voltage, and (c) the magnetic flux density calculated from the search coil voltage measured at point A in Fig. 5. The blue and red lines indicate the tangential and normal components, respectively. In Fig. 6(a), the excitation currents of the tangential and normal components exhibit the same amplitude. However, the search coil voltage did not display the coincidence between the tangential and normal components. The tangential component was larger in magnitude than that of the normal component of the search coil voltage. The measured magnetic flux density exhibited a similar result to that of the search coil voltage. This result indicates that the tangential component is dominant in the internal magnetic flux density.

Next, we discuss the internal magnetic flux density distribution in the air-core toroidal transformer. Figure 7 shows the vector field and the contour plot of the measured magnetic flux. The tangential component of the internal magnetic flux density is dominant at every measured point.

The magnetic flux density was not uniform in the tangential and normal directions. In a toroidal coil, non-uniformity in the normal direction is apparent from Eq. (2) derived from Ampere's law,

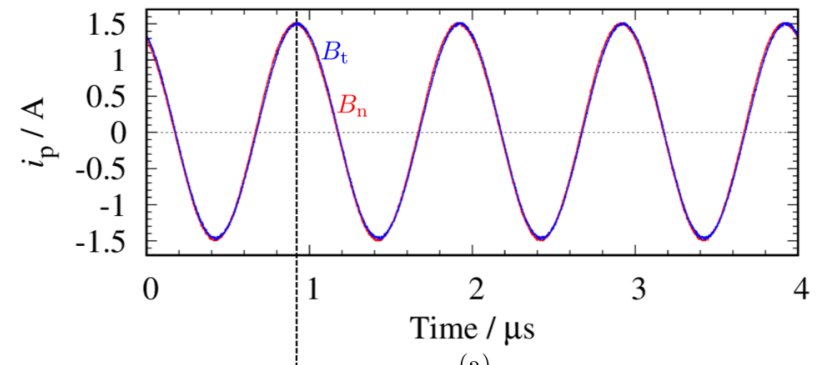

(a)

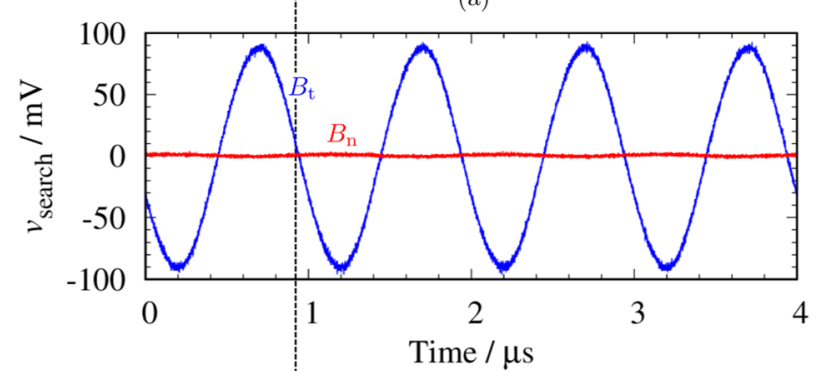

(b)

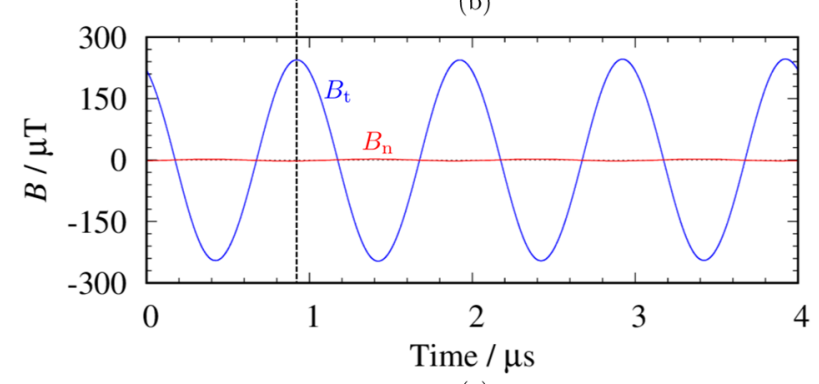

(c)

FIG. 6. Experimentally obtained waveforms: (a) excitation current $i_{p}$, (b) search

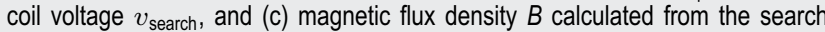
coil voltage. Blue line shows tangential components and red line shows normal components.

$$
B=\frac{\mu_{0} \mathrm{NI}}{2 \pi r},
$$

where $\mu_{0}$ denotes the permeability in a vacuum, $\mathrm{N}$ denotes the number of turns in the toroidal coil, I denotes the excitation current, and $r$ denotes the distance from the center of the toroidal coil. Equation (2) implies that the magnetic flux density decreases with increasing distance from the center of a toroidal coil. In Fig. 7, the inner magnetic flux densities were clearly larger than the outer magnetic flux densities. The experimental results were qualitatively consistent with Eq. (2). Therefore, the non-uniformity of the magnetic flux density in the normal direction is caused by the toroidal winding structure.

Subsequently, we consider the non-uniformity of the magnetic flux density in the tangential direction. With a non-existent leakage flux in the air-core toroidal transformer, the magnetic flux density in the tangential direction would be uniform. Therefore, revealing the relationship between the winding structure and the nonuniformity of the magnetic flux density in the tangential direction is important. 


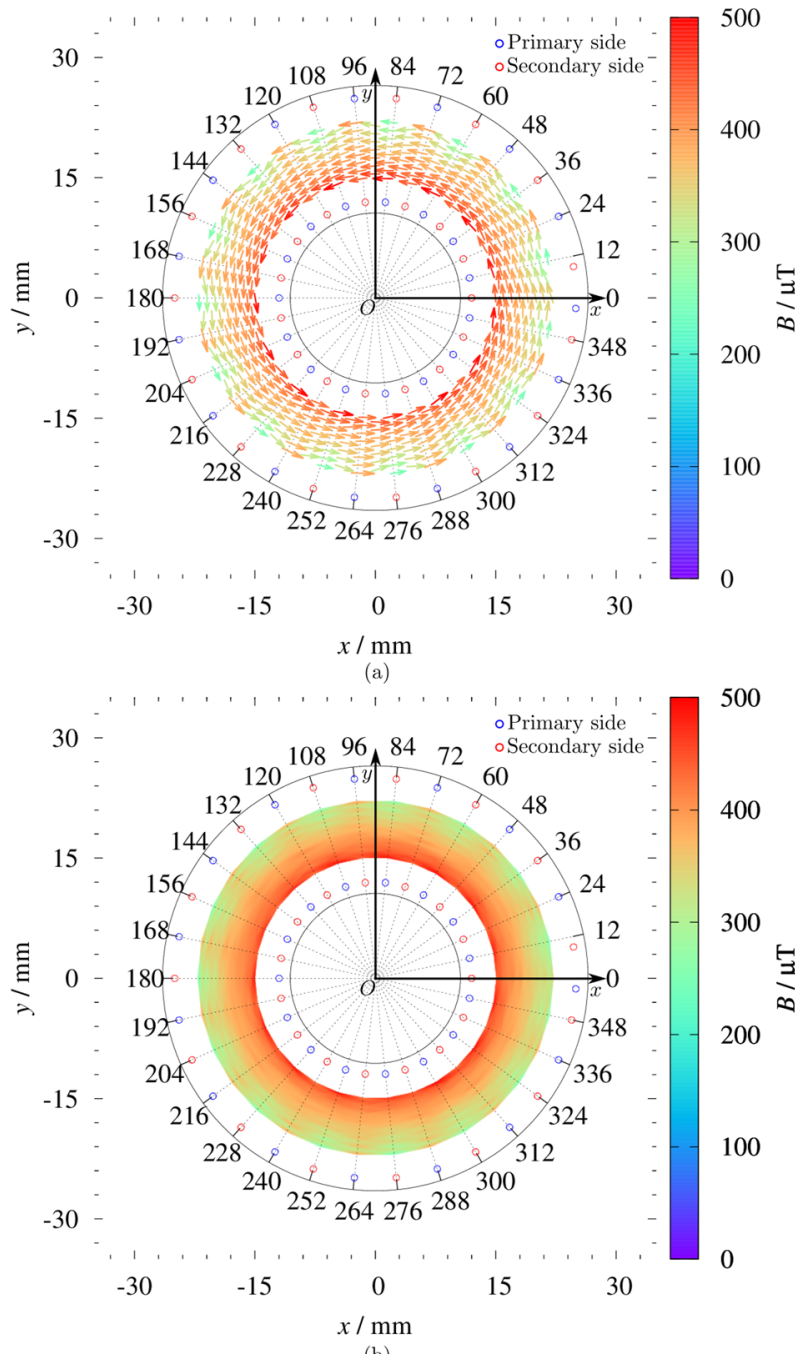

(b)

FIG. 7. Experimentally obtained internal magnetic flux density distribution in the air-core toroidal transformer: (a) vector field and (b) contour plot. Numbers around the circle denote angle. Blue circles denote primary side contact probes and red circles denote secondary side contact probes.

Figure 8 shows the magnetic flux density as a function of angle in the air-core toroidal transformer. The physical relationship between the air-core toroidal transformer structure and the radius plotted in Fig. 8 is shown in Fig. 9. The magnetic flux density changed periodically with a spatial period of $24^{\circ}$, except in Fig. 8(c). The air-core toroidal transformer is wound with a spatial period of $24^{\circ}$ because the number of turns in the primary and secondary windings is 15 turns each. Therefore, the spatial period of the nonuniformity in the tangential direction is consistent with the structure of the air-core toroidal transformer.

The spatial phases of non-uniformity show a $180^{\circ}$ difference between the outer side [Figs. 8(a) and 8(b)] and the inner side [Fig. 8(d)] of the air-core toroidal transformer. The structure of the

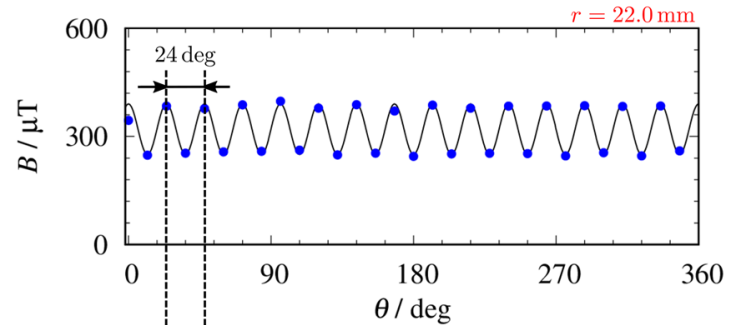

(a)

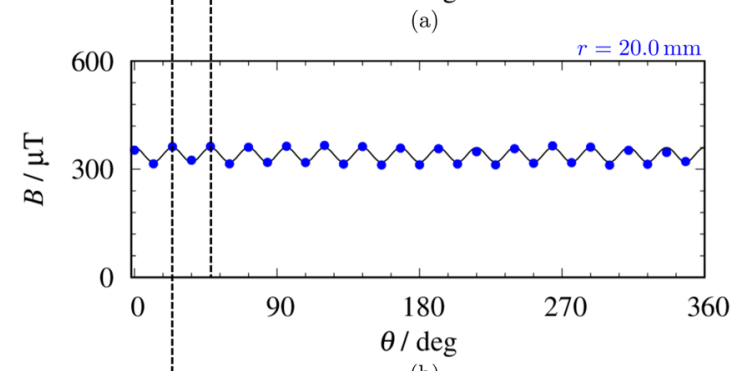

(b)

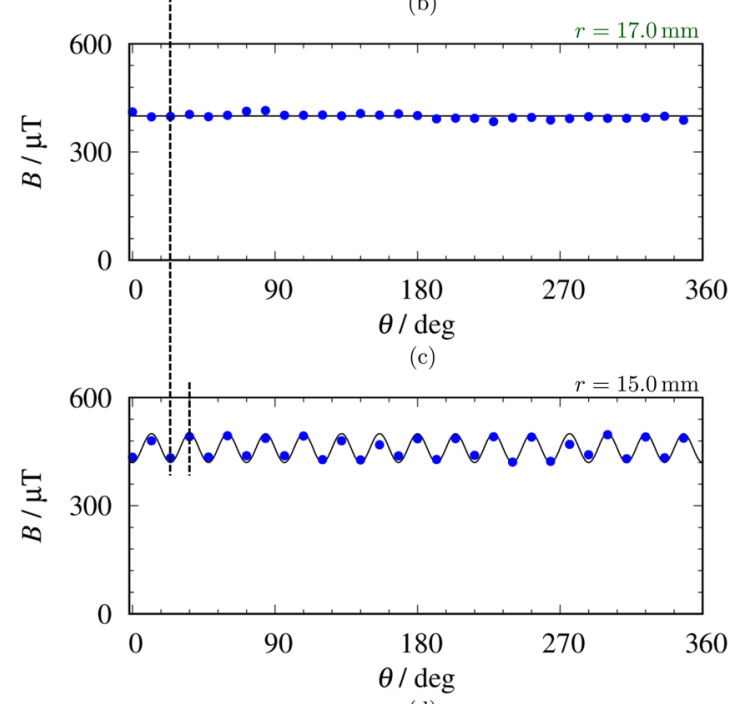

(d)

FIG. 8. Non-uniformity in the magnetic flux density in the tangential direction for: (a) $r=22.0 \mathrm{~mm}$, (b) $r=20.0 \mathrm{~mm}$, (c) $r=17.0 \mathrm{~mm}$, and (d) $r=15.0 \mathrm{~mm}$ in order to compare experimentally obtained internal magnetic flux densities as function of angle in the air-core toroidal transformer.

air-core toroidal transformer has a $180^{\circ}$ difference in the spatial phase between the inner and outer sides. The spatial phase difference of the non-uniformity displayed in the results is consistent with the structure of the air-core toroidal transformer. The structure of the air-core toroidal transformer causes the non-uniformity in the internal magnetic flux density in the tangential direction.

\section{B. Verification of non-uniformity in the internal magnetic flux density distribution}

Herein, we verify the non-uniformity exhibited in the internal magnetic flux density distribution through a comparison with a numerical estimation. We assumed that the current distribution 


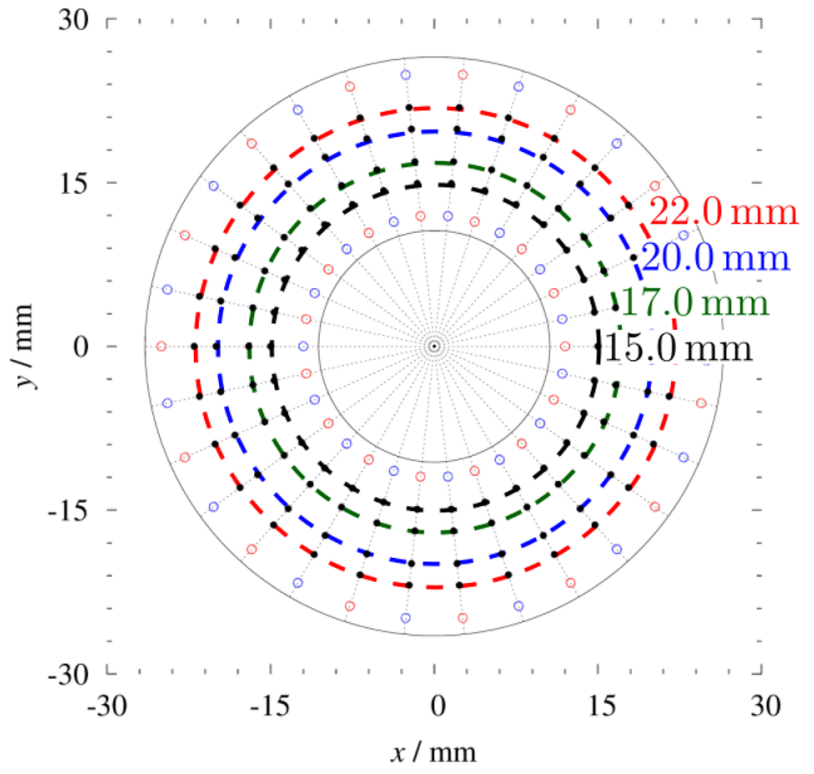

FIG. 9. Relationship between the air-core toroidal transformer structure and the radius plotted in Fig. 8.

in the air-core toroidal transformer could be represented by a set of straight conductors. The magnetic flux density of a straight conductor at a point $\mathrm{P}$ is given by Eq. (3) from the Biot-Savart law,

$$
B=\frac{\mu_{0} \mathrm{I}}{4 \pi r}(\cos \alpha+\cos \beta),
$$

where $\mu_{0}$ denotes the permeability in a vacuum, I denotes the excitation current, and $r$ denotes the distance between the straight conductor and point P. The values of $\alpha$ and $\beta$ are defined in Fig. 10. Due to the air-core configuration, the internal magnetic flux density distribution becomes the sum of the magnetic flux density provided by each straight conductor. The detailed calculation is shown in Appendix B.

Figure 11 shows the contour plot of the internal magnetic flux density distribution obtained by the numerical estimation. The

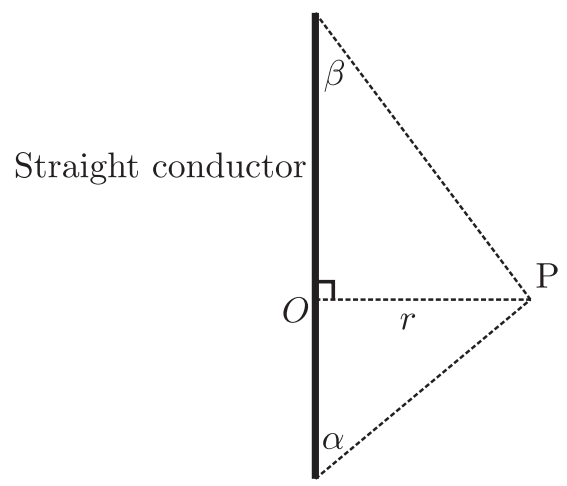

FIG. 10. Definition of $\alpha$ and $\beta$.

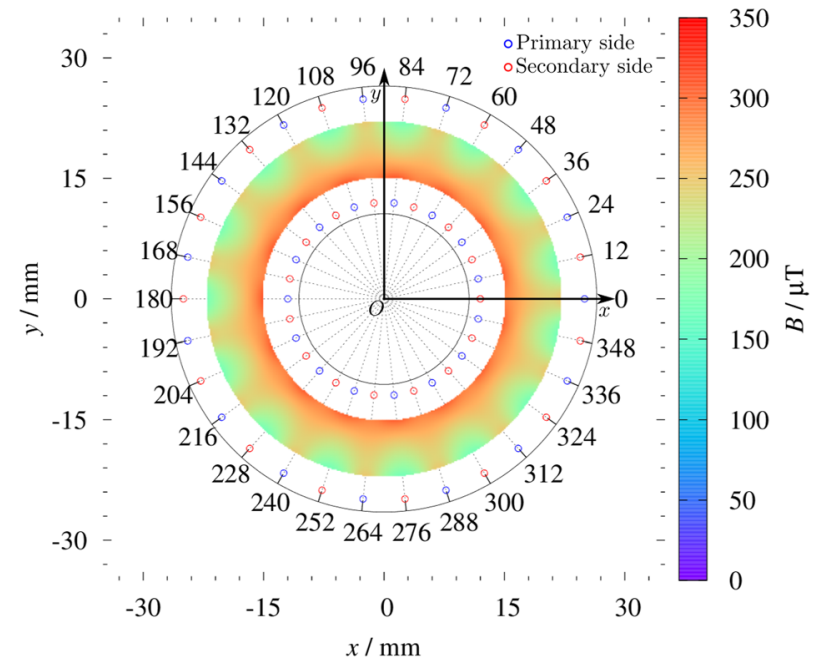

FIG. 11. Numerically obtained internal magnetic flux density in the air-core toroida transformer. Numbers around the circle denote angle. Blue circles denote primary side contact probes and red circles denote secondary side contact probes.

internal magnetic flux density in Fig. 11 showed qualitatively similar features to Fig. 7(b). There is a discrepancy between the measured and modeled magnitudes of the magnetic flux density. This is due to the manufacturing accuracy of the search coil. In order to discuss the magnetic flux density quantitatively, the area-turn product of the search coil must inevitably be calibrated by a Helmholtz coil. ${ }^{17,18}$ On the other hand, it is of greater importance to investigate whether the spatial difference in the measured magnetic flux density corresponds to the winding structure. The relative value becomes a more critical factor for choosing the winding structure, considering the magnetic flux density. Therefore, it is necessary to verify the relative value of the magnetic flux density.

Figure 12 shows the experimentally and numerically obtained magnetic flux densities as a function of angle in the air-core toroidal transformer. Blue points show the experimental results, while the red points display the results of the numerical estimation. In order to compare the relative values, the results of the numerical estimation were multiplied by 1.5 . The relative values of the experimental result and the numerical estimation were in good agreement for the spatial frequency as well as for the normalized amplitude at every radius value. This result supports the validity of the non-uniformity in the magnetic flux density obtained by the proposed measurement method.

This result also indicates that the current distribution flowing through the windings corresponds to the obtained magnetic flux density. The internal magnetic flux density distribution in this paper does not depend on the excitation waveform, as long as there is a current flow along the winding. When the current distribution changes for some reason, such as the impacts of stray capacitances, the magnetic flux density also changes. Therefore, it is possible to estimate the impact of parasitic capacitance in power converters using a comparison of the magnetic flux densities obtained by applying the proposed method to the operating air-core toroidal transformer. 




(a)

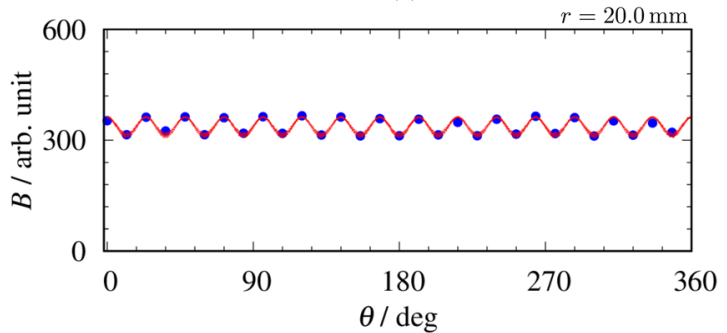

(b)

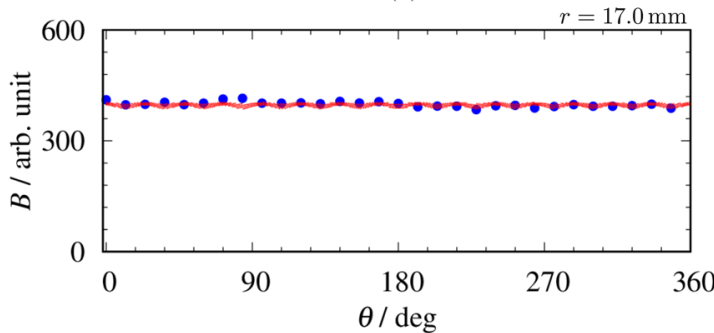

(c)

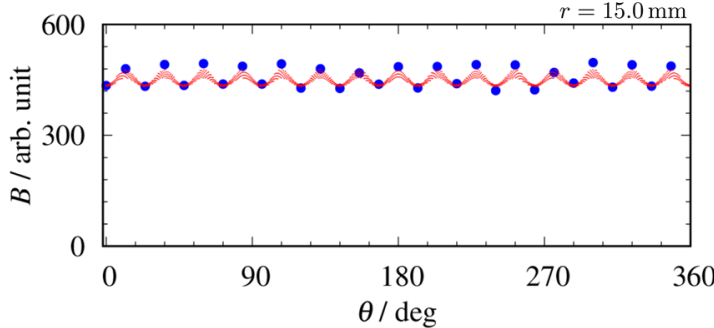

(d)

FIG. 12. Comparison of experimental results and numerical estimation for internal magnetic flux density as a function of angle in the air-core toroidal transformer for: (a) $r=22.0 \mathrm{~mm}$, (b) $r=20.0 \mathrm{~mm}$, (c) $r=17.0 \mathrm{~mm}$, and (d) $r=15.0 \mathrm{~mm}$. Blue points denote experimental results and red points denote the results of numerical estimation. Results of the numerical estimation were multiplied by 1.5 to compare relative values.

\section{CONCLUSION}

This paper proposed a measurement method for the internal magnetic flux density distribution in an air-core toroidal transformer and measured the distribution under a $1 \mathrm{MHz}$ sinusoidal excitation. The magnetic flux density was found to be non-uniform in the air-core toroidal transformer. We verified the non-uniformity of the distribution through a comparison of the experimental results to the numerical estimation by the Biot-Savart law. We conclude that the magnetic flux density obtained by the proposed method is directly linked to the structure of the air-core toroidal transformer. By analyzing the non-uniformity in the magnetic flux density, we can estimate parasitic elements, such as the stray capacitances in the air-core toroidal transformer.

\section{ACKNOWLEDGMENTS}

This work was supported by JST-OPERA Program Grant No. JPMJOP1841, Japan.

\section{APPENDIX A: FREQUENCY DEPENDENCE OF THE MEASURED MAGNETIC FLUX DENSITY WITH THE SEARCH COIL}

This appendix discusses the frequency dependence of the measured magnetic flux density based on the frequency response of the search coil. Figure 13 shows the frequency response of the fabricated search coil. The frequency response was measured with an impedance analyzer (Keysight Technologies, 4294A). As shown in Fig. 13(a), the impedance exhibits a linear response to frequencies. The phase was approximately $90^{\circ}$ from $1 \mathrm{MHz}$ to $100 \mathrm{MHz}$. Therefore, the fabricated search coil acts as a magnetic component in that frequency range.

Figure 14 shows the measured magnetic flux density in various excitation frequencies. The blue line indicates the measured magnetic flux density at $100 \mathrm{kHz}$, the red line at $1 \mathrm{MHz}$, and the green line at $10 \mathrm{MHz}$. In the excitation at $10 \mathrm{MHz}$, we used another bipolar power supply (NF CORPORATION, HSA4101), which can operate up to $10 \mathrm{MHz}$. This is because the bipolar power supply (HSA4011) described in Sec. II can only apply a sinusoidal wave in the range of frequency up to $1 \mathrm{MHz}$. The time axis is normalized by excitation frequencies to compare them. The magnetic flux density at $10 \mathrm{MHz}$ was identical to measured waveforms at $1 \mathrm{MHz}$. This is because the search coil acts as a magnetic component at $10 \mathrm{MHz}$, as mentioned

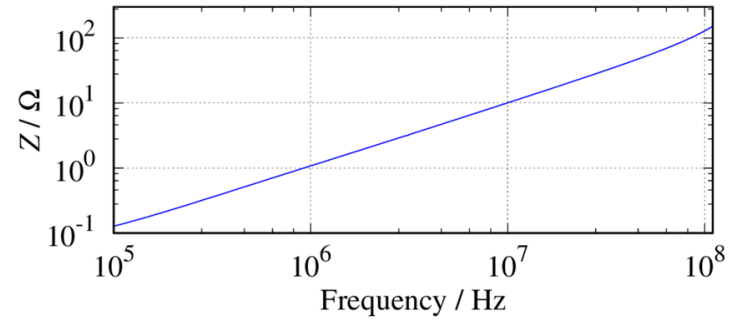

(a)

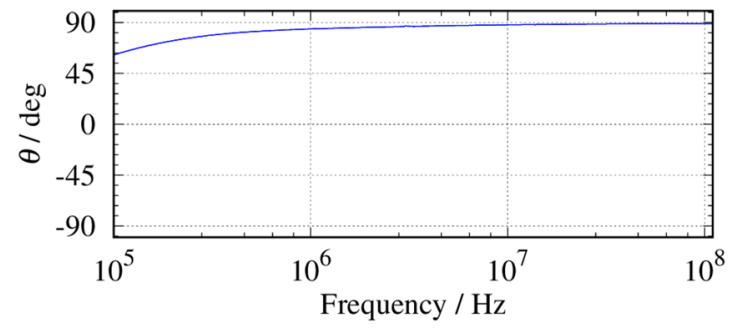

(b)

FIG. 13. Frequency response of the search coil: (a) impedance and (b) phase. 


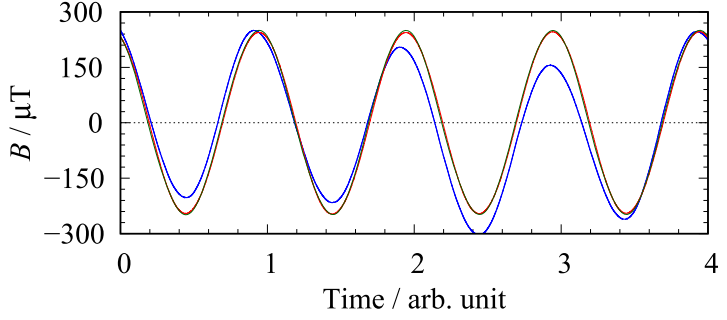

FIG. 14. Comparison of the measured magnetic flux density in various excitation frequencies. Blue line denotes the measured magnetic flux density at $100 \mathrm{kHz}$, red line at $1 \mathrm{MHz}$, and green line at $10 \mathrm{MHz}$. The time axis is normalized by excitation frequencies to compare them.

above, while the measured waveforms at $100 \mathrm{kHz}$ were not identical to them. The reason is that the isolated probe cannot measure the search coil output voltage correctly at several megavolts as the frequency response. Therefore, results at $1 \mathrm{MHz}$ ensure the validity of the measurement results from $1 \mathrm{MHz}$ to $100 \mathrm{MHz}$.

\section{APPENDIX B: MAGNETIC FIELD ESTIMATION BY THE BIOT-SAVART LAW}

First, we describe the method for estimating the magnetic flux density generated by the contact probe. The contact probe provides $x$ and $y$ components of the magnetic flux density because the contact probe and the estimation plane intersect vertically. Figure 15 shows a schematic of the relationship between the current flowing along the contact probe and the magnetic flux density. The magnitude of the magnetic flux density $B$ at $\left(x_{0}, y_{0}\right)$ can be estimated by Eq. (3). Based on this equation and Fig. 15, $x$ and $y$ components of the magnetic flux density can be determined by Eqs. (B1a) and (B1b), when the estimated position is in the first quadrant:

$$
B_{x}=-\frac{\left|\frac{y_{0}}{x_{0}}\right|}{\sqrt{1+\left(\frac{y_{0}}{x_{0}}\right)^{2}}} B \quad\left(x_{0}>0, y_{0}>0\right),
$$

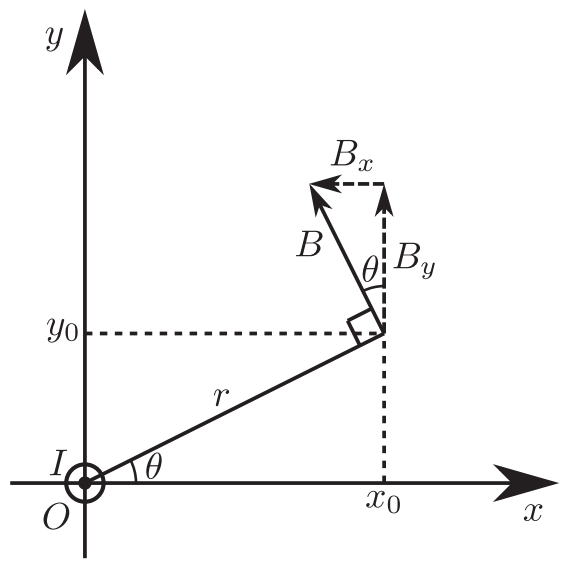

FIG. 15. Schematic of the relationship between the current flowing along the contact probe and the magnetic flux density.

$$
B_{y}=\frac{1}{\sqrt{1+\left(\frac{y_{0}}{x_{0}}\right)^{2}}} B \quad\left(x_{0}>0, y_{0}>0\right) .
$$

In other quadrants, we can obtain the magnetic flux density by modifying the sign of Eqs. (B1a) and (B1b) based on Fig. 15. By translating the obtained magnetic flux density to the position of the contact probe, the magnetic flux density can be obtained at each point.

Next, we describe the method of estimating the magnetic flux density generated by the PCB. The PCB pattern has a fan-like shape. For simplicity, this pattern is approximated as a straight conductor. The PCB provides $x, y$, and $z$ components of the magnetic flux density because the PCB and the estimation plane do not intersect vertically. We consider the magnetic flux density as provided by a straight conductor parallel to the $\mathrm{x}$-axis. The magnetic flux density exists only in $y$ and $z$ components under this condition. The magnetic flux density of the $y$ component is given by Eq. (B2),

$$
B_{y}=\frac{B}{\sqrt{1+\left(\frac{y_{0}}{z_{0}}\right)^{2}}} .
$$

Therefore, by translating the obtained magnetic flux density to the position of the contact probe, the magnetic flux density is obtained at each point.

\section{REFERENCES}

${ }^{1}$ R. C. N. Pilawa-Podgurski, A. D. Sagneri, J. M. Rivas, D. I. Anderson, and D. J. Perreault, IEEE Trans. Power Electron. 24, 1654 (2009).

${ }^{2}$ Z.-L. Zhang, Z. Dong, X.-W. Zou, and X. Ren, IEEE Trans. Power Electron. 32, 6197 (2017).

${ }^{3}$ Z. Zhang and K. D. T. Ngo, IET Power Electron. 10, 1138 (2017).

${ }^{4}$ D. J. Perreault, J. Hu, J. M. Rivas, Y. Han, O. Leitermann, R. C. N. PilawaPodgurski, A. Sagneri, and C. R. Sullivan, in 2009 Twenty-Fourth Annual IEEE Applied Power Electronics Conference and Exposition (IEEE, 2009), pp. 1-14.

${ }^{\mathbf{5}}$ T. Kimoto, Jpn. J. Appl. Phys., Part 1 54, 040103 (2015).

${ }^{6}$ J. A. Cooper, M. R. Melloch, R. Singh, A. Agarwal, and J. W. Palmour, IEEE Trans. Electron Devices 49, 658 (2002).

${ }^{7}$ J. Millán, P. Godignon, X. Perpiñá, A. Pérez-Tomás, and J. Rebollo, IEEE Trans. Power Electron. 29, 2155 (2014).

${ }^{8}$ K. Nagaoka, K. Chikamatsu, A. Yamaguchi, K. Nakahara, and T. Hikihara, IEICE Electron. Express 12, 20150285 (2015).

${ }^{9}$ S. Orlandi, B. A. Allongue, G. Blanchot, S. Buso, F. Faccio, C. A. Fuentes, M. Kayal, S. Michelis, and G. Spiazzi, IEEE Trans. Power Electron. 26, 1837 (2011).

${ }^{10}$ C. Liu, D. Mandal, Z. Yao, M. Sun, J. Todsen, B. Johnson, S. Kiaei, and B. Bakkaloglu, IEEE Trans. Circuits Syst. II 66, 1003 (2019).

${ }^{11}$ W. Liang, L. Raymond, and J. Rivas, IEEE Trans. Power Electron. 31, 52 (2016).

${ }^{12}$ H. Thanh Le, Y. Nour, A. Han, F. Jensen, Z. Ouyang, and A. Knott, IEEE J. Emerging Sel. Top. Power Electron. 6, 604 (2018).

${ }^{13}$ R. Wang, H. F. Blanchette, M. Mu, D. Boroyevich, and P. Mattavelli, IEEE Trans. Power Electron. 28, 4568 (2013).

${ }^{14}$ K. B. McEachron, J. Am. Inst. Electr. Eng. 41, 281 (1922).

${ }^{15}$ A. E. Kennelly and P. L. Alger, Trans. Am. Inst. Electr. Eng. XXXVI, 1113 (1917).

${ }^{16}$ Y. Ohbuchi, T. Urabe, and Y. Sakurai, IEEE Trans. Magn. 7, 899 (1971).

${ }^{17}$ R. C. Phillips and E. B. Turner, Rev. Sci. Instrum. 36, 1822 (1965).

${ }^{18}$ E. L. Bronaugh, in Proceedings of International Symposium on Electromagnetic Compatibility (IEEE, 1995), pp. 72-76. 